\title{
Prostatic Secretion Alteration
}

National Cancer Institute

\section{Source}

National Cancer Institute. Prostatic Secretion Alteration. NCI Thesaurus. Code C40937.

Prostatic Secretion Alteration involves a qualitative change in existing activities involved in release into seminal fluid of acid phosphatase, citric acid, and proteolytic enzymes that liquefy coagulated semen, from the accessory sex gland of the male reproductive system that surrounds the neck of the bladder and urethra (prostate gland). 